\title{
Quantum mechanical look at the radioactive-like decay of metastable dark energy
}

\author{
Marek Szydłowski $^{1,2, a} \mathbb{0}$, Aleksander Stachowski ${ }^{1, b}$, Krzysztof Urbanowski $^{3, c}$ \\ ${ }^{1}$ Astronomical Observatory, Jagiellonian University, Orla 171, 30-244 Kraków, Poland \\ ${ }^{2}$ Mark Kac Complex Systems Research Centre, Jagiellonian University, Łojasiewicza 11, 30-348 Kraków, Poland \\ ${ }^{3}$ Institute of Physics, University of Zielona Góra, Prof. Z. Szafrana 4a, 65-516 Zielona Gora, Poland
}

Received: 6 November 2017 / Accepted: 13 December 2017 / Published online: 23 December 2017

(C) The Author(s) 2017. This article is an open access publication

\begin{abstract}
We derive the Shafieloo, Hazra, Sahni and Starobinsky (SHSS) phenomenological formula for the radioactive-like decay of metastable dark energy directly from the principles of quantum mechanics. To this aim we use the Fock-Krylov theory of quantum unstable states. We obtain deeper insight on the decay process as having three basic phases: the phase of radioactive decay, the next phase of damping oscillations, and finally the phase of power-law decay. We consider the cosmological model with matter and dark energy in the form of decaying metastable dark energy and study its dynamics in the framework of non-conservative cosmology with an interacting term determined by the running cosmological parameter. We study the cosmological implications of metastable dark energy and estimate the characteristic time of ending of the radioactive-like decay epoch to be $2.2 \times 10^{4}$ of the present age of the Universe. We also confront the model with astronomical data which show that the model is in good agreement with the observations. Our general conclusion is that we are living in the epoch of the radioactive-like decay of metastable dark energy which is a relict of the quantum age of the Universe.
\end{abstract}

\section{Introduction}

We follow Krauss and Dent's paper and apply the FockKrylov theory of unstable quantum states to analyze a cosmological scenario with decaying dark energy [1-5]. For this purpose we extend the Shafieloo, Hazra, Sahni and Starobinsky (SHSS) model of metastable dark energy with radioactive-like decay [6] and we give physical motivation arising directly from quantum mechanics for phenomenolog-

\footnotetext{
${ }^{a}$ e-mail: marek.szydlowski@uj.edu.pl

be-mail: aleksander.stachowski@doctoral.uj.edu.pl

c e-mail: K.Urbanowski@if.uz.zgora.pl
}

ical formulas for SHSS model of the dark energy. We replace the radioactive, classical physics constant decay rate by the decay rate derived using the Fock-Krylov theory of unstable quantum states.

As a result we obtain a logistic-type radiative decay of dark energy, which is followed by the much slower decay process than the radioactive one, known as the quantum Zeno effect. Within such an approach we find the energy of the system in the unstable state and the decay rate. The rigorous results show that these quantities both are time dependent. We find the exact analytical expression for them assuming that the density of the energy distribution, $\omega(E)$, in the unstable state has the Breit-Wigner form. Using these results we also find the late times asymptotic expressions of these quantities. Then we assume that the dark energy density decays and that this is a quantum process. Starting from these assumptions we use the derived decay rate to analyze the decay process of the dark energy density.

We study the cosmological implications of the derived formula for decaying dark energy in the framework of flat FRW cosmology. We find an extension of the standard cosmological model in the form of an interacting cosmology in which the energy-momentum tensor is not conserved due to the interaction between the dark energy and dark matter by energy transfer.

We consider the problem if the decay of the running lambda term can solve the cosmological constant problem and how it can modify the canonical scaling law of energy density for dark matter. We also test the model by astronomical observations.

Our statistical analysis gives the best fit values of the density parameters for each component of the decaying vacuum of the dark energy. Testing the model with observational data we have found that dark energy can decay in three distinguished ways: exponentially, by damping oscillation and in power-law decay. We show that the main contribution to the 
decay of the metastable vacuum is the dark energy decay of an exponential type and this type of decay dominates up to $2.2 \times 10^{4} T_{0}$, where $T_{0}$ is the present age of the Universe. Our calculations show that the exponential decay has only an intermediate character and will be replaced in the future evolution of the Universe by an oscillation decay and decay of $1 / t^{2}$ type. From the estimation of the model parameters we see that the decay half life should be much larger than the age of the Universe.

Today modern cosmology has the methodological status of some effective theory, which is described very well by current astronomical observations in terms of dark matter and dark energy. However, there are many open problems related to the unknown nature of dark energy. The cosmological parameter is a good effective description of the accelerating phase of the current Universe, but we do not understand why the today value of this parameter is so small in comparison with its value in the early Universe.

We look for an alternative cosmological model to supersede the $\Lambda \mathrm{CDM}$ model, the present standard cosmological model. Our main motivation is to check if the model considered in the next sections is able to solve the cosmological constant problem. In this paper, we consider the case when the cosmological constant parameter results from the assumption that the vacuum energy is given by the fundamental theory [7].

We assume quantum mechanics as a fundamental theory, which determines the cosmological parameters and explain how the cosmological parameters change during the cosmic evolution. The discussion of the cosmological constant problem is included in Refs. [7-20].

Krauss and Dent [1] analyzed the properties of the false vacuum state form the point of view of the quantum theory of decay processes. They assumed that the decay process of metastable vacuum is a quantum decay process realized as the transition from the state corresponding to the metastable (false) vacuum state to the state corresponding to the lowest energy of the Universe (that is, to the true vacuum state) and thus that this process can be described using the standard quantum formalism usually used to describe the decay of excited atomic levels or unstable particles. They used the Fock-Krylov theory of unstable quantum states [2-5]. One of the famous results of this theory is the proof that unstable quantum systems cannot decay exponentially at very late times and that in such a late time regime any decay process must run slower than any exponentially decreasing function of time [4]. Model calculations show that survival probability exhibits inverse power-law behavior at these times. Krauss and Dent [1] analyzing a false vacuum decay pointed out that in eternal inflation, many false vacuum regions can survive up to much later than the times when the exponential decay law holds. They formulated the hypothesis that some false vacuum regions survive well up to the cross-over time $T$ or later, where the cross-over time, $T$, is the time when contributions of the exponential and late time non-exponential parts of the survival probability are of the same order. They gave a simple explanation of such an effect. It may occur even though the regions of false vacua by assumption should decay exponentially, and gravitational effects force space in a region that has not decayed yet to grow exponentially fast. Such a cosmological scenario may be realized if the lifetime of the metastable vacuum state or the dark energy density is much, much shorter than the age of the Universe. It should be of order of times of the age of the inflationary stage of the Universe.

The possibility that our Universe (or some regions in our Universe) were able to survive up to times longer that the cross-over time $T$ should be considered seriously was concluded by Krauss and Dent's analysis [1]. This is impossible within the standard approach of calculations of the decay rate $\Gamma$ for the decaying vacuum state [21-25]. Calculations performed within this standard approach cannot lead to a correct description of the evolution of the Universe with false vacuum in all cases when the lifetime of the false vacuum state is so short that its survival probability exhibits an inverse powerlaw behavior at times comparable with the age of the Universe. This conclusion is valid not only when the dark energy density and its late time properties are related to the transition of the Universe from the false vacuum state to the true vacuum, but also when the dark energy is formed by unstable "dark particles". In both cases the decay of the dark energy density is the quantum decay process and only the formalism based on the Fock-Krylov theory of unstable quantum states and used by Krauss and Dent [1] is able to describe correctly such a situation. Note that Landim and Abdalla built a model of metastable dark energy, in which the observed vacuum energy is the value of the scalar potential at the false vacuum [26].

Models with metastable dark energy have recently been discussed in the context of the explanation of the $H_{0}$ tension problem [27]. Our model is a quantum generalization of Shafieloo et al.'s model [6] and contains a phase of radioactive-like decay valid in the context of solving this problem. Shafieloo et al. considered three different ways of dark energy decay. In our paper, we investigate the second way of the decay into dark matter. The models of the decay of the dark energy analyzed in [6] can be a useful tool for numerically testing decay processes discussed in [1] and for analyzing the properties of the decaying dark energy at times $t>T$. Namely, Shafieloo et al. [6] analyzed the properties of the model of the time evolution of the dark energy. Their model assumes a "radioactive decay" scheme for decaying dark energy in which the present value of the dark energy density, $\rho_{\mathrm{DE}}\left(t_{0}\right)$, is related to its value at an earlier instant of time, $\rho_{\mathrm{DE}}(t)$, by 
$\rho_{\mathrm{DE}}(t)=\rho_{\mathrm{DE}}\left(t_{0}\right) \times \exp \left[-\Gamma\left(t-t_{0}\right)\right] \equiv \rho_{\mathrm{DE}}\left(t-t_{0}\right)$,

where the only free parameter is the decay rate $\Gamma$. Shafieloo et al. [6] derived this equation from the fundamental equation of the theory of radioactive decays,

$\dot{\rho}_{\mathrm{DE}}(t)=-\Gamma \rho_{\mathrm{DE}}(t)$

(see Eqs. (2.1) and (2.2) in [6]). These equations are known from the Rutherford theory of the decay of radioactive elements. Rutherford deriving these equations assumed that the number decaying radioactive elements at a given instant of time is proportional to a number of these elements at this moment of time [28-31] as in Eq. (2). So the Rutherford equations and thus also Eqs. (1)-(2) are the classical physics equations.

In the context of Eqs. (1)-(2) one may ask what $\rho_{\mathrm{DE}}(t)$ is built from that decays according to radioactive decay law? For physicists the only reasonable explanation for this problem is the assumption that $\rho_{\mathrm{DE}}(t)$ describes the energy of an extremely huge number of particles occupying a volume $V_{0}$ at the initial instant of time $t_{0}$ and decaying at later times. Of course when such particles can be considered as classical particles, then this process can be described using the classical radioactive decay law. Unfortunately the process of the creation of the Universe is not a classical physics process, but it is a quantum process and particles or states of the system created during such a process exhibit quantum properties and are subject to the laws of quantum physics. The same concerns $\rho_{\mathrm{DE}}(t)$ generated by quantum fluctuations or excitations of a quantum scalar field, which can be described as excited metastable states of this field and the process of their decay is a quantum process. Therefore, as a quantum decay process it exhibits at late times completely different properties than the classical radioactive decay process, as pointed out by Krauss and Dent. Simply, if $\rho_{\mathrm{DE}}(t)$ is related to the extremely huge number of metastable states (excitations of the scalar field or its fluctuations) generated at $t_{0}$ in a volume $V_{0}$, it is very likely that many of them can be found undecayed at times longer than the cross-over time $T$. All this suggests that Eqs. (1) and (2) may not be used when one wants to describe such a processes.

It seems that a reasonable way to make these equations suitable for description of quantum decay processes is to replace the quantity (the decay rate) $\Gamma$ appearing in Eqs. (1) and (2) by a corresponding decay rate derived using the quantum theory of unstable systems. The decay rate $\Gamma$ used in Eqs. (1) and (2) is constant in time but the decay rate derived within the quantum theory is constant to a very good approximation only at the so-called "canonical decay regime" of times $t$ (that is, when the quantum decay law has the exponential form, i.e. when $t<T$ ) and at times $t$ much later than $T$ it tends to zero as $1 / t$ when time $t$ tends to infin- ity (see, e.g., [32]). This means that the decay process of an unstable quantum system is slower and slower for sufficiently late time, which was also pointed out in [1]. This and other properties of the quantum decay process seem to be important when considering the cosmological inflationary and late time (much later than the inflationary regime of times) processes including transition processes of the dark energy density from its early time extremely large values to its present small value. Therefore we need quantities characterizing the decay processes of unstable quantum systems.

The paper is organized as follows. Section 2 contains a brief introduction to the problems of unstable states and a description of quantities characterizing such states, which are used in the next sections. In Sect. 3 we analyze a possibility to describe metastable dark energy considering it as an unstable quantum system. Section 4 contains a discussion of the cosmological equations with decaying dark energy according to the quantum mechanical decay law, and the results of the numerical calculations are presented in graphical form. In Sect. 5 we present a statistical analysis. Section 6 contains the conclusions.

\section{Preliminaries: unstable quantum states}

The properties of unstable quantum systems are characterized by their survival probability (decay law). The survival probability can be found knowing the initial unstable state $|\phi\rangle \in \mathcal{H}(\mathcal{H}$ is the Hilbert space of states of the considered system) of the quantum system, which was prepared at the initial instant $t_{0}$. The survival probability, $\mathcal{P}(t)$, of this state $|\phi\rangle$ decaying in vacuum equals $\mathcal{P}(t)=|A(t)|^{2}$, where $A(t)$ is the probability amplitude of finding the system at the time $t$ in the rest frame $\mathcal{O}_{0}$ in the initial unstable state $|\phi\rangle, A(t)=\langle\phi \mid \phi(t)\rangle$. Here $|\phi(t)\rangle$ is the solution of the Schrödinger equation for the initial condition $\left|\phi\left(t_{0}\right)\right\rangle=|\phi\rangle$, which has the following form:

$i \hbar \frac{\partial}{\partial t}|\phi(t)\rangle=\mathfrak{H}|\phi(t)\rangle$.

Here $|\phi\rangle,|\phi(t)\rangle \in \mathcal{H}$, and $\mathfrak{H}$ denotes the total self-adjoint Hamiltonian for the system considered. The spectrum of $\mathfrak{H}$ is assumed to be bounded from below: $E_{\min }>-\infty$ is the lower bound of the spectrum $\sigma_{c}(\mathfrak{H})=\left[E_{\min },+\infty\right)$ of $\left.\mathfrak{H}\right)$. Using the basis in $\mathcal{H}$ built from normalized eigenvectors $|E\rangle, \quad E \in$ $\sigma_{c}(\mathfrak{H})$ of $\mathfrak{H}$ and using the expansion of $|\phi\rangle$ in this basis one can express the amplitude $A(t)$ as the following Fourier integral:

$A(t) \equiv A\left(t-t_{0}\right)=\int_{E_{\min }}^{\infty} \omega(E) e^{-\frac{i}{\hbar} E\left(t-t_{0}\right)} \mathrm{d} E$,

where $\omega(E)=\omega(E)^{*}$ and $\omega(E)>0($ see $[2,3,5])$. Note that from the normalization condition $\mathcal{P}(0) \equiv|A(0)|^{2}=1$ 
it follows that $\int_{E_{\min }}^{\infty} \omega(E) \mathrm{d} E=1$, which means that in the case of unstable states $\omega(E)$ is an absolutely integrable function. The consequence of this property is the conclusion following from the Riemann-Lebesgue lemma: we need to have $|A(t)| \rightarrow 0$ as $t \rightarrow \infty$. All these properties are the essence of the so-called Fock-Krylov theory of unstable states $[2,3,5]$. So within this approach the amplitude $A(t)$, and thus the decay law $\mathcal{P}(t)$ of the unstable state $|\phi\rangle$, are completely determined by the density of the energy distribution $\omega(E)$ for the system in this state [2,3] (see also [4,5,33-37]. (This approach is also applicable in quantum field theory models $[38,39]$.)

Note that in fact the amplitude $A(t)$ contains information as regards the decay law $\mathcal{P}(t)$ of the state $|\phi\rangle$, that is, as regards the decay rate $\Gamma_{\phi}$ of this state, as well as the energy $E_{\phi}$ of the system in this state. This information can be extracted from $A(t)$. It can be done using the rigorous equation governing the time evolution in the subspace of unstable states, $\mathcal{H}_{\|} \ni|\phi\rangle_{\|} \equiv|\phi\rangle$. Such an equation follows from the Schrödinger equation (3) for the total state space $\mathcal{H}$.

Using the Schrödinger equation (3) one finds that for the problem considered

$i \hbar \frac{\partial}{\partial t}\langle\phi \mid \phi(t)\rangle=\langle\phi|\mathfrak{H}| \phi(t)\rangle$.

From this relation one can conclude that the amplitude $A(t)$ satisfies the following equation:

$i \hbar \frac{\partial A(t)}{\partial t}=h(t) A(t)$

where

$h(t)=\frac{\langle\phi|\mathfrak{H}| \phi(t)\rangle}{A(t)} \equiv \frac{i \hbar}{A(t)} \frac{\partial A(t)}{\partial t}$

and $h(t)$ is the effective Hamiltonian governing the time evolution in the subspace of unstable states $\mathcal{H}_{\|}=\mathbb{P} \mathcal{H}$, where $\mathbb{P}=|\phi\rangle\langle\phi|$ (see [32] and also [41,42] and the references therein). The subspace $\mathcal{H} \ominus \mathcal{H}_{\|}=\mathcal{H}_{\perp} \equiv \mathbb{Q} \mathcal{H}$ is the subspace of decay products. Here $\mathbb{Q}=\mathbb{I}-\mathbb{P}$. One meets the effective Hamiltonian $h(t)$ when one starts with the Schrödinger equation for the total state space $\mathcal{H}$ and looks for the rigorous evolution equation for a distinguished subspace of states $\mathcal{H}_{\|} \subset \mathcal{H}[32,37]$. In general, $h(t)$ is a complex function of time and in the case of $\mathcal{H}_{\|}$of dimension two or more the effective Hamiltonian governing the time evolution in such a subspace it is a non-hermitian matrix $H_{\|}$or a non-hermitian operator. We have

$h(t)=E_{\phi}(t)-\frac{i}{2} \Gamma_{\phi}(t)$, and $E_{\phi}(t)=\Re[h(t)]$ and $\Gamma_{\phi}(t)=-2 \Im[h(t)]$, are the instantaneous energy (mass) $E_{\phi}(t)$ and the instantaneous decay rate, $\Gamma_{\phi}(t)[32,41,42]$. (Here $\Re(z)$ and $\Im(z)$ denote the real and imaginary parts of $z$, respectively.) The quantity $\Gamma_{\phi}(t)=-2 \mathfrak{\Im}[h(t)]$ is interpreted as the decay rate because it satisfies the definition of the decay rate used in quantum theory: $\frac{\Gamma_{\phi}(t)}{\hbar} \stackrel{\text { def }}{=}-\frac{1}{\mathcal{P}(t)} \frac{\partial \mathcal{P}(t)}{\partial t}$. Using (7) it is easy to check that

$$
\begin{aligned}
\frac{\Gamma_{\phi}(t)}{\hbar} & \equiv-\frac{1}{\mathcal{P}(t)} \frac{\partial \mathcal{P}(t)}{\partial t}=-\frac{1}{|A(t)|^{2}} \frac{\partial|A(t)|^{2}}{\partial t} \\
& \equiv-\frac{2}{\hbar} \Im[h(t)] .
\end{aligned}
$$

The use of the effective Hamiltonian $h(t)$ leads to the following form of the solutions of Eq. (6):

$A(t)=e^{-i \frac{t}{\hbar} \overline{h(t)}} \equiv e^{-i \frac{t}{\hbar}\left(\overline{E_{\phi}(t)}-\frac{i}{2} \overline{\Gamma_{\phi}(t)}\right)}$,

where $\overline{h(t)}$ is the average effective Hamiltonian $h(t)$ for the time interval $[0, t]: \overline{h(t)} \stackrel{\text { def }}{=} \frac{1}{t} \int_{0}^{t} h(x) \mathrm{d} x$ (averages $\overline{E_{\phi}(t)}, \overline{\Gamma_{\phi}(t)}$ are defined analogously). Within a rigorous treatment of the problem it is straightforward to show that the basic assumptions of the quantum theory guarantee that (see, e.g. [32])

$\lim _{t \rightarrow \infty} \Gamma_{\phi}(t)=0$ and $\lim _{t \rightarrow \infty} \overline{\Gamma_{\phi}(t)}=0$.

These results are rigorous. For $\overline{E_{\phi}(t)}$ one can show that $\lim _{t \rightarrow \infty} \overline{E_{\phi}(t)}=E_{\min }$ (see [43]).

Equations (6) and (7) are convenient when the density $\omega(E)$ is given and one wants to find the instantaneous energy $E_{\phi}(t)$ and decay rate $\Gamma_{\phi}(t)$ : Inserting $\omega(E)$ into (4) one obtains the amplitude $A(t)$ and then using (7) one finds the $h(t)$ and thus $E_{\phi}(t)$ and $\Gamma_{\phi}(t)$. In the general case the density $\omega(E)$ possesses properties analogous to the scattering amplitude, i.e., it can be decomposed into a threshold factor, a pole-function $P(E)$ with a simple pole and a smooth form factor $F(E)$. We have $\omega(E)=\Theta\left(E-E_{\min }\right)\left(E-E_{\min }\right)^{\alpha_{l}} P(E) F(E)$, where $\alpha_{l}$ depends on the angular momentum $l$ through $\alpha_{l}=\alpha+l$ (see Eq. (6.1) in [5]), $0 \leq \alpha<1)$ and $\Theta(E)$ is a step function: $\Theta(E)=0$ for $E \leq 0$ and $\Theta(E)=1$ for $E>0$. The simplest choice is to take $\alpha=0, l=0, F(E)=1$ and to assume that $P(E)$ has the Breit-Wigner (BW) form of the energy distribution density. (The mentioned BreitWigner distribution was found when the cross-section of slow neutrons was analyzed [44].) It turns out that the decay curves obtained in this simplest case are very similar in form to the curves calculated for the above described more general $\omega(E)$ (see [33] and the analysis in [5]). So to find the most typical properties of the decay process it is suf- 
ficient to make the relevant calculations for $\omega(E)$ modeled by the Breit-Wigner distribution of the energy density: $\omega(E) \equiv \omega_{\mathrm{BW}}(E) \stackrel{\text { def }}{=} \frac{N}{2 \pi} \Theta\left(E-E_{\min }\right) \frac{\Gamma_{0}}{\left(E-E_{0}\right)^{2}+\left(\frac{\Gamma_{0}}{2}\right)^{2}}$, where $N$ is a normalization constant. The parameters $E_{0}$ and $\Gamma_{0}$ correspond to the energy of the system in the unstable state and its decay rate at the exponential (or canonical) regime of the decay process. $E_{\min }$ is the minimal (the lowest) energy of the system. Inserting $\omega_{\mathrm{BW}}(E)$ into Eq. (4) for the amplitude $A(t)$ after some algebra one finds that

$A(t)=A\left(t-t_{0}\right)=\frac{N}{2 \pi} e^{-\frac{i}{\hbar} E_{0} t} I_{\beta}\left(\frac{\Gamma_{0}\left(t-t_{0}\right)}{\hbar}\right)$,

where

$I_{\beta}(\tau) \stackrel{\text { def }}{=} \int_{-\beta}^{\infty} \frac{1}{\eta^{2}+\frac{1}{4}} e^{-i \eta \tau} \mathrm{d} \eta$.

Here $\tau=\frac{\Gamma_{0}\left(t-t_{0}\right)}{\hbar} \equiv \frac{t-t_{0}}{\tau_{0}}, \tau_{0}$ is the lifetime, $\tau_{0}=\frac{\hbar}{\Gamma_{0}}$, and $\beta=\frac{E_{0}-E_{\min }}{\Gamma_{0}}>0$. (The integral $I_{\beta}(\tau)$ can be expressed in terms of the integral-exponential function [40-42]; for a definition, see $[45,46]$.)

Note that the more convenient is to use $t^{\prime}=\left(t-t_{0}\right)$ in (12), (13) or (4) and in a formula of this type, or to assume that $t_{0}=0$ in all formulas of this type, because this does not change the results of calculations but makes them easier. So from this point on we will assume that $t_{0}=0$.

Next using this $A(t)$ given by Eqs. (12), (13) and Eq. (7) defining the effective Hamiltonian $h_{\phi}(t)$ one finds that within the Breit-Wigner (BW) model considered

$h(t)=E_{0}+\Gamma_{0} \frac{J_{\beta}\left(\frac{\Gamma_{0} t}{\hbar}\right)}{I_{\beta}\left(\frac{\Gamma_{0} t}{\hbar}\right)}$,

where

$J_{\beta}(\tau)=\int_{-\beta}^{\infty} \frac{x}{x^{2}+\frac{1}{4}} e^{-i x \tau} \mathrm{d} x$.

Working within the BW model and using $J_{\beta}(\tau)$ one should remember that $J_{\beta}(0)$ is undefined $\left(\lim _{\tau \rightarrow 0} J_{\beta}(\tau)=\infty\right)$. Simply within the model defined by the Breit-Wigner distribution of the energy density, $\omega_{\mathrm{BW}}(E)$, the expectation value of $\mathfrak{H}$, that is, $\langle\phi|\mathfrak{H}| \phi\rangle$, is not finite. So the whole consideration based on the use of $J_{\beta}(\tau)$ is valid only for $\tau>0$.

It is relatively simple to find the analytical form of $J_{\beta}(\tau)$ using the following identity:

$J_{\beta}(\tau) \equiv i \frac{\partial I_{\beta}(\tau)}{\partial \tau}$.

We need to know the energy of the system in the unstable state $|\phi\rangle$ considered and its decay rate. The instantaneous energy $E_{\phi}(t)$ of the system in the unstable state $|\phi\rangle$ has the following form within the $\mathrm{BW}$ model considered:

$E_{\phi}(t)=\Re[h(t)]=E_{0}+\Gamma_{0} \Re\left[\frac{J_{\beta}\left(\frac{\Gamma_{0} t}{\hbar}\right)}{I_{\beta}\left(\frac{\Gamma_{0} t}{\hbar}\right)}\right]$,

whereas the instantaneous decay rate looks as follows:

$$
\begin{aligned}
\Gamma_{\phi}(\tau) & =-2 \Im[h(t)]=-2 \Gamma_{0} \Im\left[\frac{J_{\beta}(\tau)}{I_{\beta}(\tau)}\right] \\
& \equiv-2 \Gamma_{0} \Im\left[\frac{J_{\beta}\left(\frac{\Gamma_{0} t}{\hbar}\right)}{I_{\beta}\left(\frac{\Gamma_{0} t}{\hbar}\right)}\right] .
\end{aligned}
$$

It is relatively simple to find the asymptotic expressions $I_{\beta} \tau$ and $J_{\beta}(\tau)$ for $\tau \rightarrow \infty$ directly from (13) and (15) using, e.g., the method of integration by parts. We have for $\tau \rightarrow \infty$

$$
\begin{aligned}
I_{\beta}(\tau) \simeq & \frac{i}{\tau} \frac{e^{i \beta \tau}}{\beta^{2}+\frac{1}{4}}\left\{-1+\frac{2 \beta}{\beta^{2}+\frac{1}{4}} \frac{i}{\tau}\right. \\
& \left.+\left[\frac{2}{\beta^{2}+\frac{1}{4}}-\frac{8 \beta^{2}}{\left(\beta^{2}+\frac{1}{4}\right)^{2}}\right]\left(\frac{i}{\tau}\right)^{2}+\cdots\right\}
\end{aligned}
$$

and

$$
\begin{aligned}
J_{\beta}(\tau) \simeq & \frac{i}{\tau} \frac{e^{i \beta \tau}}{\beta^{2}+\frac{1}{4}}\left\{\beta+\left[1-\frac{2 \beta^{2}}{\beta^{2}+\frac{1}{4}}\right] \frac{i}{\tau}\right. \\
& \left.+\frac{\beta}{\beta^{2}+\frac{1}{4}}\left[\frac{8 \beta^{2}}{\beta^{2}+\frac{1}{4}}-6\right]\left(\frac{i}{\tau}\right)^{2}+\cdots\right\} .
\end{aligned}
$$

These two last asymptotic expressions allow one to find for $\tau \rightarrow \infty$ the asymptotic form of the ratio $\frac{J_{\beta}(\tau)}{I_{\beta}(\tau)}$ used in Eqs. (14), (17) and (18), having a much simpler form than asymptotic expansions for $I_{\beta}(\tau)$ and $J_{\beta}(\tau)$. One finds that, for $\tau \rightarrow \infty$,

$\frac{J_{\beta}(\tau)}{I_{\beta}(\tau)} \simeq-\beta-\frac{i}{\tau}-\frac{2 \beta}{\beta^{2}+\frac{1}{4}} \frac{1}{\tau^{2}}+\cdots$.

Starting from this asymptotic expression and Eq. (17) one finds, e.g. that, for $t \rightarrow \infty$,

$\left.E_{\phi}(t)\right|_{t \rightarrow \infty} \simeq E_{\min }-2 \frac{E_{0}-E_{\min }}{\left|h_{\phi}^{0}-E_{\min }\right|^{2}}\left(\frac{\hbar}{t}\right)^{2}$,

where $h_{\phi}^{0}=E_{0}-\frac{i}{2} \Gamma_{0}$, and

$\left.\Gamma_{\phi}(t)\right|_{t \rightarrow \infty} \simeq 2 \Gamma_{0} \frac{1}{\tau}+\cdots=2 \frac{\hbar}{t}+\cdots$.

The last two relations are valid for $t>T$, where $T$ denotes the cross-over time, i.e. the time when exponential and late 
time inverse power-law contributions to the survival amplitude become comparable.

\section{Metastable dark energy with a decay law from quantum mechanics}

Note that the model described by Eqs. (1)-(2) is the classical physics model and therefore it cannot be applied directly when one would like to follow Krauss and Dent and to consider the decay of the dark energy density $\rho_{\mathrm{DE}}(t)$ as the quantum decay process. For example, the late time effects discussed in [1] can never occur in the SHSS model. The simplest way to extend models considered in [6] so that they might be used to describe the decay of $\rho_{\mathrm{DE}}(t)$ as a quantum process seems to be a replacement of the classical decay rate $\Gamma$ in Eqs. (1), (2) by the decay rate $\Gamma_{\phi}(t) / \hbar$ appearing in the quantum theoretical considerations. It is because the classical decay rate $\Gamma_{\text {class }}=\Gamma$ corresponds to the quantum physics decay rate $\Gamma_{\text {quant }}=\Gamma_{\phi}(t)$ divided by $\hbar$ (that is, to $\Gamma_{\phi}(t) / \hbar$ ) and using $\Gamma_{\phi}(t)$ one can insert it into Eq. (2) to obtain

$\dot{\rho}_{\mathrm{DE}}(t)=-\frac{1}{\hbar} \Gamma_{\phi}(t) \rho_{\mathrm{DE}}(t)$,

instead of the classical fundamental equation of the radioactive decays theory. In fact this equation is a simple improvement of models discussed in [6], and it can be considered as the use of quantum corrections in the models mentioned. In such a case Eq. (1) takes the following form:

$$
\begin{aligned}
\rho_{\mathrm{DE}}(t) & =\rho_{\mathrm{DE}}\left(t_{0}\right) \times \exp \left[-\frac{t}{\hbar} \overline{\Gamma_{\phi}(t)}\right] \\
& \equiv \rho_{\mathrm{DE}}\left(t_{0}\right) \times \exp \left[-\frac{1}{\hbar} \int_{t_{0}}^{t} \Gamma_{\phi}(x) \mathrm{d} x\right],
\end{aligned}
$$

where $\Gamma_{\phi}(t)$ is given by Eq. (18) and $\overline{\Gamma_{\phi}(t)} \stackrel{\text { def }}{=} \frac{1}{t} \int_{t_{0}}^{t} \Gamma_{\phi}(x) \mathrm{d} x$ is the average decay rate for the time interval $[0, t]$. These relations, replacing Eq. (1), contain quantum corrections connected with the use of the quantum theory decay rate.

Note that using the identity (9) and Eq. (12) one can rewrite Eq. (26) as follows:

$\rho_{\mathrm{DE}}(t) \equiv \frac{N^{2}}{4 \pi^{2}} \rho_{\mathrm{DE}}\left(t_{0}\right)\left|I_{\beta}\left(\frac{\Gamma_{0}\left(t-t_{0}\right)}{\hbar}\right)\right|^{2}$,

which can make simpler numerical calculations.

Now in order to obtain analytical or numerical results having Eqs. (24)-(26) one needs a quantum mechanical model of the decay process, that is, one needs $\omega(E)$ (see (4)). We begin our considerations using the Breit-Wigner model analyzed in the previous section. Inserting $\Gamma_{\phi}(t)$ given by (18) into Eq. (24), or Eqs. (25) and (26) we can analyze the decay process of $\rho_{\mathrm{DE}}(t)$. One can notice that performing the calculations, e.g. using the Breit-Wigner model, it is more convenient to use Eq. (27) with $I_{\beta}(t)$ given by Eq. (13) than using Eqs. (25) and (26) with $\Gamma_{\phi}(t)$ given by Eq. (18).

Note that one of the parameters appearing in the quantum mechanical formula (18) for $\Gamma_{\phi}(t)$ is $\Gamma_{0}$. This parameter can be eliminated if we notice that $\beta=\frac{E_{0}-E_{\min }}{\Gamma_{0}}>0$. Hence $\Gamma_{0} \equiv \frac{E_{0}-E_{\min }}{\beta}$, and therefore one can rewrite (18) as

$\Gamma_{\phi}(\tau)=-2 \frac{E_{0}-E_{\min }}{\beta} \Im\left[\frac{J_{\beta}(\tau)}{I_{\beta}(\tau)}\right]$,

or

$\Gamma_{\phi}(\tau)=-2 \frac{\frac{E_{0}}{V_{0}}-\frac{E_{\min }}{V_{0}}}{\beta} V_{0} \Im\left[\frac{J_{\beta}(\tau)}{I_{\beta}(\tau)}\right]$,

where $V_{0}$ is the volume of the system at $t=t_{0}$. We have $\frac{E_{0}}{V_{0}}=$ $\rho_{\mathrm{DE}}^{q f t} \stackrel{\text { def }}{=} \rho_{D E}^{0}$ and $\frac{E_{\min }}{V_{0}}=\rho_{\text {bare }}$, (where $\rho_{D E}^{q f t}$ is the energy density calculated using quantum field theory methods), so Eq. (29) can be rewritten as follows:

$\Gamma_{\phi}(\tau)=-2 \frac{\rho_{\mathrm{DE}}^{0}-\rho_{\text {bare }}}{\beta} V_{0} \Im\left[\frac{J_{\beta}(\tau)}{I_{\beta}(\tau)}\right]$.

The parameter $\tau$ used in (28)-(30) denotes time $t$ measured in lifetimes as mentioned after Eq. (13): $\tau=\frac{t}{\tau_{0}}$. Using the parameter $\beta$ the lifetime $\tau_{0}$ can be expressed as follows: $\tau_{0}=$ $\frac{\beta}{\rho_{\mathrm{DE}}^{0}-\rho_{\text {bare }}} \frac{\hbar}{V_{0}}$.

The asymptotic form (23) indicates one of the main differences between the SHSS model and our improvement of this model. Namely, from Eq. (1) it follows that

$\lim _{t \rightarrow \infty} \rho_{\mathrm{DE}}(t)=0$.

From (1) one sees that $\rho_{\mathrm{DE}}(t)$ is an exponentially decreasing function of time.

It is interesting to consider a more general form of the energy density,

$\tilde{\rho}_{\mathrm{DE}}(t)=\rho_{\mathrm{DE}}(t)-\rho_{\text {bare }}$,

where $\rho_{\text {bare }}=$ const is the minimal value of the dark energy density. Inserting the density $\tilde{\rho}_{\mathrm{DE}}(t)$ into Eq. (1) one concludes that $\rho_{\mathrm{DE}}(t)$ tends to $\rho_{\text {bare }}$ exponentially fast as $t \rightarrow \infty$.

Let us see now what happens when we insert $\tilde{\rho}_{\mathrm{DE}}(t)$ into our Eq. (24) and consider only the asymptotic behavior of $\rho_{\mathrm{DE}}(t)$ for times $t \geq T_{0} \gg T$. In such a case inserting the late time asymptotic expression of Eq. (23) into Eq. (24) one finds for very late times $t>T_{0}$ that

$\ln \frac{\tilde{\rho}_{\mathrm{DE}}(t)}{\tilde{\rho}_{\mathrm{DE}}\left(T_{0}\right)}=\ln \left(\frac{t}{T_{0}}\right)^{-2}$, 
that is, for $t>T_{0} \gg T$,

$\rho_{\mathrm{DE}}(t) \simeq \rho_{\text {bare }}+D \frac{1}{t^{2}}$

where $D=$ const. Note that the same result follows directly from (27) when one inserts there $A(t)$ given by Eq. (12) and uses the asymptotic expression of Eq. (19) for $I_{\beta}(\tau)$, which shows that our approach is self-consistent. The result (34) means that quantum corrections do not allow $\rho_{\mathrm{DE}}(t)$ to tend to $\rho_{\text {bare }}$ exponentially fast when $t \rightarrow \infty$, but $\rho_{\mathrm{DE}}(t)$ must tend to $\rho_{\text {bare }}$ as $1 / t^{2}$, for $t \rightarrow \infty$, which is in the full agreement with our earlier results, presented, e.g., in $[20,47-50]$. So in fact, as one can see, the SSHS model is the classical physics approximation of the model discussed in our papers mentioned, where the cosmological parametrization resulting from the quantum mechanical treatment of unstable systems was used.

\section{Cosmological equations}

We introduce our model as the covariant theory with the interaction term [51]. We consider the flat cosmological model (the constant curvature is equal zero).

The total density of energy consists of the baryonic matter $\rho_{\mathrm{B}}$, the dark matter $\rho_{\mathrm{DM}}$ and the dark energy $\rho_{\mathrm{DE}}$. We assume, for the baryonic matter and the dark matter, the equation of state for dust $\left(p_{\mathrm{B}}\left(\rho_{\mathrm{B}}\right)=0\right.$ and $\left.p_{\mathrm{DM}}\left(\rho_{\mathrm{DM}}\right)=0\right)$. Also we consider the equation of state for the dark energy to be $p_{\mathrm{DE}}\left(\rho_{\mathrm{DE}}\right)=-\rho_{\mathrm{DE}}$.

The cosmological equations such as the Friedmann and acceleration equations are found by the variation action by the metric $g_{\mu \nu}$ [51]. In consequence we get the equations

$3 H^{2}=3 \frac{\dot{a}^{2}}{a}=\rho_{\mathrm{tot}}=\rho_{\mathrm{B}}+\rho_{\mathrm{DM}}+\rho_{\mathrm{DE}}$

and

$\frac{\ddot{a}}{a}=-\frac{1}{6}\left(\rho_{\mathrm{tot}}+3 p_{\mathrm{tot}}\left(\rho_{\mathrm{tot}}\right)\right)=\rho_{\mathrm{B}}+\rho_{\mathrm{DM}}-2 \rho_{\mathrm{DE}}$,

where $H=\frac{\dot{a}}{a}$ is the Hubble function. Here, we assume $8 \pi G=c=1$.

Equations (35) and (36) give the conservation equation in the following form:

$\dot{\rho}_{\text {tot }}=-3 H\left(\rho_{\text {tot }}+p_{\text {tot }}\left(\rho_{\text {tot }}\right)\right)$

or in the equivalent form

$\dot{\rho}_{\mathrm{M}}=-3 H \rho_{\mathrm{M}}-\dot{\rho}_{\mathrm{DE}}$,

where $\rho_{\mathrm{M}}=\rho_{\mathrm{B}}+\rho_{\mathrm{DM}}$.

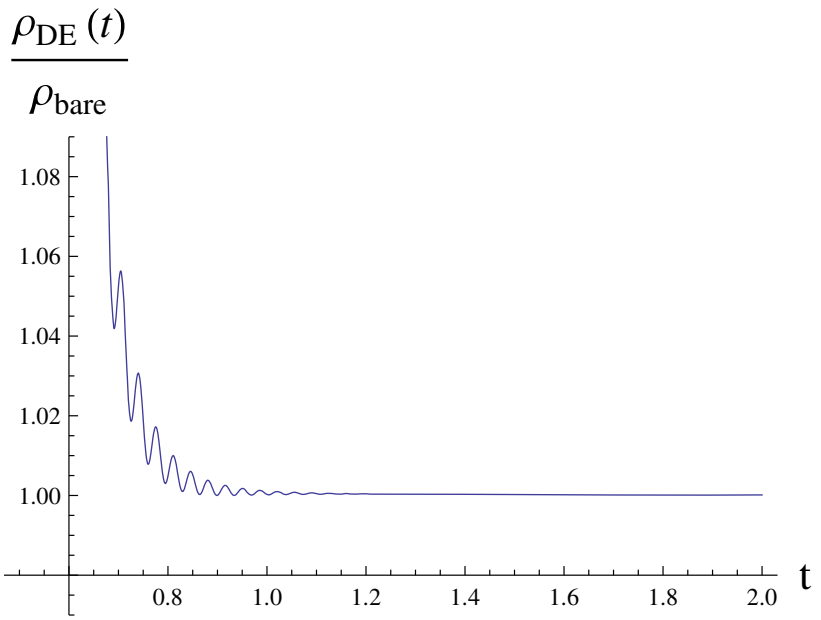

Fig. 1 The dependence $\rho_{\mathrm{DE}}(t)$ [from Eq. (40)]. For illustration we put $\beta=800, \Gamma_{0}=20 \hbar$ and $\epsilon=1000 \rho_{\text {bare }}$. The qualitative behavior of $\rho_{\mathrm{DE}}$ does not depend on $\epsilon$. The units of time $t$ are determined by the choice of units of $\Gamma_{0}$ because $\frac{\Gamma_{0} t}{\hbar}$ is dimensionless

Let $Q$ denote the interaction term. Equation (38) can be rewritten as

$\dot{\rho}_{\mathrm{b}}=-3 H \rho_{\mathrm{B}}, \quad \dot{\rho}_{\mathrm{DM}}=-3 H \rho_{\mathrm{DM}}+Q$ and $\dot{\rho}_{\mathrm{DE}}=-Q$.

If $Q>0$ then the energy flows from the dark energy sector to the dark matter sector. If $Q<0$ then the energy flows from the dark matter sector to the dark energy sector.

Figure 1 shows the diagrams of the evolution of $\rho_{\mathrm{DE}}(t)$. Note that the oscillatory phase appears in the evolution of $\rho_{\text {DE }}(t)$. Figure 2 presents the evolution of the $\bar{\Gamma}_{\phi}(t)$. At the initial period we obtain a logistic-type decay of dark energy. The period when $\bar{\Gamma}_{\phi}(t)$ grows to a plateau is characteristic for the so-called Zeno time [52]. It increases slowly about 0.0004 (the slope of this curve is 0.0001 ) with the cosmic time $t$ in the interval $(0,4)$. Then in the interval $(4,30000)$ it becomes strictly constant. This behavior justifies a radioactive approximation given in Ref. [6]. For the late time, $\bar{\Gamma}_{\phi}(t)$ approaches zero.

Using (27) we get the final formula for $\rho_{\mathrm{DE}}(t)$,

$\rho_{\mathrm{DE}}(t)=\rho_{\text {bare }}+\epsilon\left|I_{\beta}\left(\frac{\Gamma_{0} t}{\hbar}\right)\right|^{2}$,

where $\epsilon \equiv \epsilon(\beta)=\frac{\rho_{\mathrm{DE}}(0)-\rho_{\text {bare }}}{\left|I_{\beta}(0)\right|^{2}}$ measures the deviation from the $\Lambda$ CDM model $\left(I_{\beta}(0) \equiv \frac{2 \pi}{N}=\pi+2 \arctan (2 \beta)\right.$ and $\beta>0)$.

The canonical scaling law for cold dark matter should be modified. In this case

$\rho_{\mathrm{DM}}=\rho_{\mathrm{DM}, 0} a^{-3+\delta}$, 

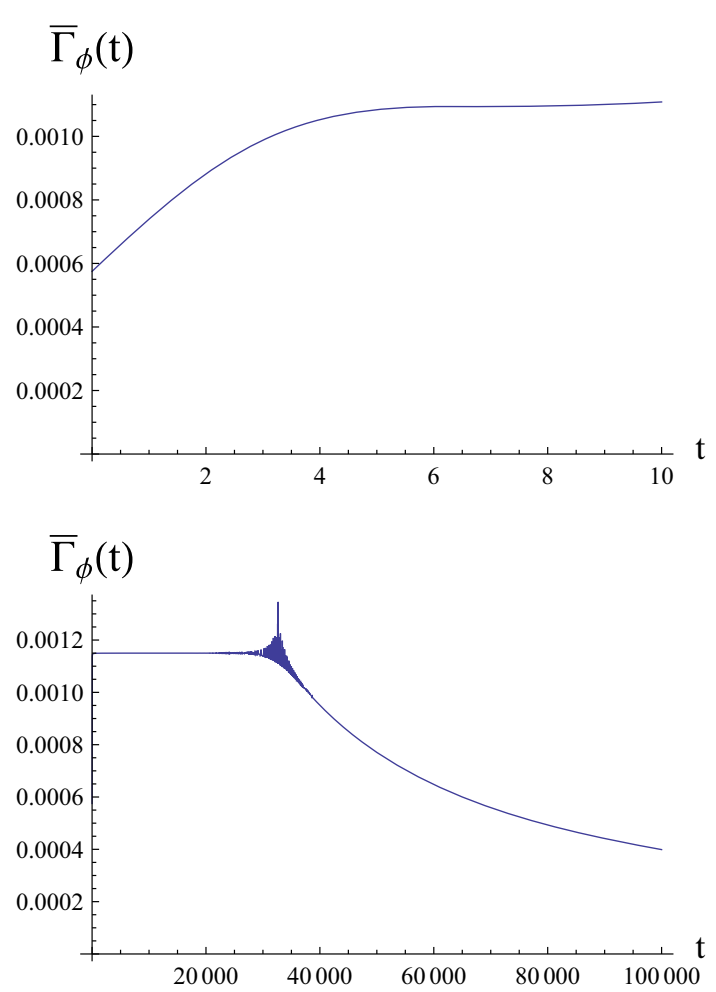

Fig. 2 The dependence $\bar{\Gamma}_{\phi}(t)$ for the best fit values (see Table 1). The upper panel presents the evolution of $\bar{\Gamma}_{\phi}(t)$ for the early Universe and the present epoch. The lower panel presents evolution of $\bar{\Gamma}_{\phi}(t)$ for the late time Universe. The cosmological time $t$ is expressed in $\frac{\mathrm{s} \times \mathrm{Mpc}}{100 \mathrm{~km}}$. In these units, the age of the Universe is equal $1.41 \frac{\mathrm{s} \times \mathrm{Mpc}}{100 \mathrm{~km}}$

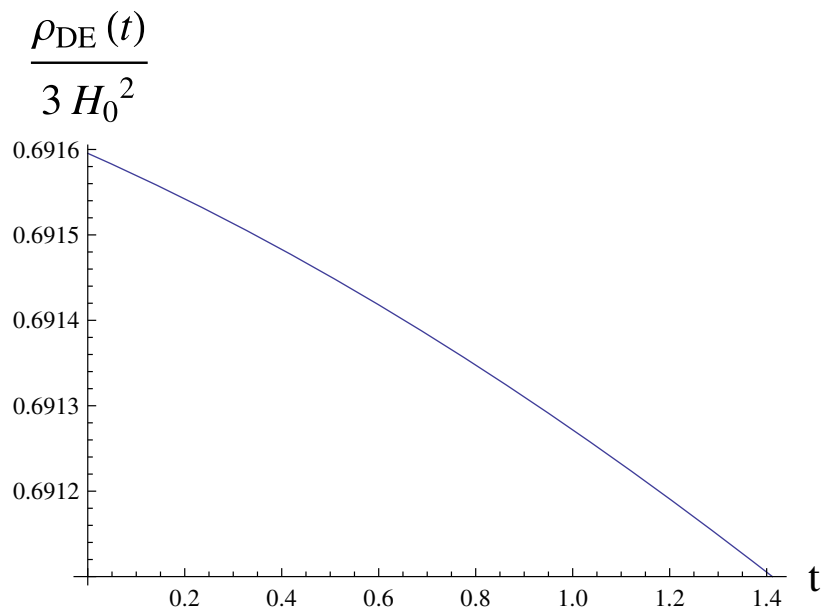

Fig. 3 The dependence $\rho_{\mathrm{DE}}(t)$ (from Eq. (40)) for the best fit value of model parameter (see Table 1). The cosmological time $t$ is expressed in $\frac{\mathrm{s} \times \mathrm{Mpc}}{100 \mathrm{~km}}$. The present epoch is for $t=1.41 \frac{\mathrm{s} \times \mathrm{Mpc}}{100 \mathrm{~km}}$. Note that, in the Planck epoch, the value of $\frac{\rho_{\mathrm{DE}}\left(t_{\mathrm{Pl}}\right)}{3 H_{0}^{2}}$ is equal to 0.6916

where $\delta=\frac{1}{\ln a} \int \frac{Q}{H \rho_{\mathrm{DM}}} d \ln a$. The dependence $\rho_{\mathrm{DE}}(t)$ [from Eq. (40)] for the best fit value of model parameter (see Table 1) is presented in Fig. 3 and the evolution of $\delta(t)$ is shown in Fig. 4.
Assuming that $\beta>0$ one obtains for $t>t_{L}=\frac{\hbar}{\Gamma_{0}} \frac{2 \beta}{\beta^{2}+\frac{1}{4}}$ (see [40]) the approximation of (40) in the following form:

$$
\begin{aligned}
& \rho_{\mathrm{DE}}(t) \approx \rho_{\text {bare }} \\
& +\epsilon\left(4 \pi^{2} e^{-\frac{\Gamma_{0}}{\hbar} t}+\frac{4 \pi e^{-\frac{\Gamma_{0}}{2 \hbar} t} \sin \left(\beta \frac{\Gamma_{0}}{\hbar} t\right)}{\left(\frac{1}{4}+\beta^{2}\right) \frac{\Gamma_{0}}{\hbar} t}+\frac{1}{\left(\left(\frac{1}{4}+\beta^{2}\right) \frac{\Gamma_{0}}{\hbar} t\right)^{2}}\right) .
\end{aligned}
$$

For the best fit value (see Table 1) $t_{L} \approx 2 T_{0}$.

From Eq. (42), it results that, for the late time, the behavior of dark energy can be described by the following formula:

$\rho_{\mathrm{DE}}(t) \approx \rho_{\text {bare }}+\frac{\epsilon}{\left(\left(\frac{1}{4}+\beta^{2}\right) \frac{\Gamma_{0}}{\hbar}\right)^{2}} \frac{1}{t^{2}}$.

This case was considered in $[53,54]$.

If we use Eq. (42) in the Friedmann equation (35), we get

$$
\begin{aligned}
3 H^{2}= & \rho_{\text {tot }}=\rho_{\mathrm{B}}+\rho_{\mathrm{DM}}+\rho_{\text {bare }}+\rho_{\text {rad.dec }} \\
& +\rho_{\text {dam.osc. }}+\rho_{\text {pow.law }},
\end{aligned}
$$

where $\rho_{\text {rad.dec. }}=4 \pi^{2} \epsilon e^{-\frac{\Gamma_{0}}{\hbar} t}$ is the radioactive-like decay part of the dark energy, $\rho_{\text {dam.osc. }}=\frac{4 \pi \epsilon e^{-\frac{\Gamma_{0}}{2 \hbar} t} \sin \left(\beta \frac{\Gamma_{0}}{\hbar} t\right)}{\left(\frac{1}{4}+\beta^{2}\right) \frac{\Gamma_{0}}{\hbar} t}$ represents the damping oscillations part of the dark energy and $\rho_{\text {pow.law }}=\frac{\epsilon}{\left(\left(\frac{1}{4}+\beta^{2}\right) \frac{\Gamma_{0}}{\hbar} t\right)^{2}}$ represents the power-law part of the dark energy. Using dimensionless parameters, $\Omega_{i}=\frac{\rho_{i}}{3 H_{0}^{2}}$, where $H_{0}$ is the present value of the Hubble constant, Eq. (44) can be rewritten as

$\frac{H^{2}}{H_{0}^{2}}=\Omega_{\mathrm{B}}+\Omega_{\mathrm{DM}}+\Omega_{\text {bare }}+\Omega_{\text {rad.dec. }}+\Omega_{\text {dam.osc. }}+\Omega_{\text {pow.law }}$.

If the radioactive-like decay dominates then one can define the e-folding time $\lambda$ and half life time $T_{1 / 2}=\lambda \ln 2=\frac{\hbar \ln 2}{\Gamma_{0}}$.

The evolution of $\Omega_{\text {rad.dec. }}, \Omega_{\text {dam.osc. }}, \Omega_{\text {pow.law }}$ with respect to time, for the best fit value (see Table 1), is presented in Fig. 5.

In the moment when the period of the radioactive-like

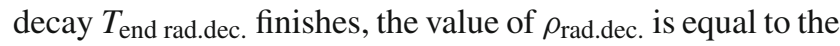
value of $\rho_{\text {dam.osc. }}$. It leads us to the condition

$4 \pi^{2} \epsilon e^{-\frac{\Gamma_{0}}{\hbar} t}=\frac{4 \pi \epsilon e^{-\frac{\Gamma_{0}}{2 \hbar} t} \sin \left(\beta \frac{\Gamma_{0}}{\hbar} t\right)}{\left(\frac{1}{4}+\beta^{2}\right) \frac{\Gamma_{0}}{\hbar} t}$,

or, after simplifying,

$\pi e^{-\frac{\Gamma_{0}}{2 \hbar} t}=\frac{\sin \left(\beta \frac{\Gamma_{0}}{\hbar} t\right)}{\left(\frac{1}{4}+\beta^{2}\right) \frac{\Gamma_{0}}{\hbar} t}$. 


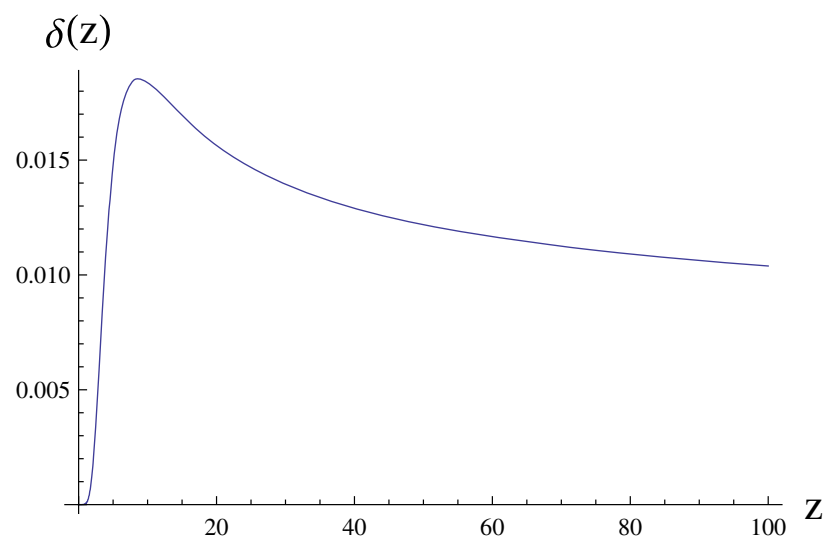

Fig. 4 A diagram of the evolution of $\delta(z)$, where $z$ is redshift. For illustration we put $\beta=800, \Gamma_{0}=20 \hbar$ and $\epsilon=1000 \rho_{\text {bare }}$. The function $\delta(z)$ reaches the maximum for $z=z_{0}$, which is a solution of equation $\delta\left(z_{0}\right)=\frac{Q\left(z_{0}\right)}{H\left(z_{0}\right) \rho_{\mathrm{DM}}\left(z_{0}\right)}$

Equation (47) has infinitely many solutions but $T_{\text {end rad.dec. is }}$ equal to the least positive real solution of (47) because the period of the radioactive-like decay is before the period of the damping oscillation decay.

Searching for the value of $T_{\text {end rad.dec. }}$ can be simplified by using of the upper envelope of oscillations of $\rho_{\text {dam.osc. }}$, which is given by

$e_{\text {upper }}(t)=\frac{4 \pi \epsilon e^{-\frac{\Gamma_{0}}{2 \hbar} t}}{\left(\frac{1}{4}+\beta^{2}\right) \frac{\Gamma_{0}}{\hbar} t}$.

Then we get an approximation of Eq. (47) in the form $\rho_{\text {rad.dec. }}=e_{\text {upper }}$ or after simplifying

$\pi e^{-\frac{\Gamma_{0}}{2 \hbar} t}=\frac{1}{\left(\frac{1}{4}+\beta^{2}\right) \frac{\Gamma_{0}}{\hbar} t}$.

The solution of Eq. (49) gives us the approximated value of $T_{\text {end rad.dec. }}$.

Note that a solution of Eq. (49) cannot be less than the value of $T_{\text {end rad.dec. having subtracted the value of one period }}$ of oscillation of $\rho_{\text {dam.osc. }}$ (i.e., $T_{\text {dam.osc. }}=\frac{2 \pi \hbar}{\beta \Gamma_{0}}$ ) and cannot be greater than the value of $T_{\text {end rad.dec. In consequence for }}$ $\beta>29$, the error of the approximation is less than $1 \%$. The dependence $T_{\text {end rad.dec. }}(\beta)$ is presented in Fig. 6 .

From the statistical analysis (see Sect. 5), we have the best fit values of $\Gamma_{0} / \hbar=0.00115$ and $\beta=\frac{1}{\alpha}-1=799$ (see Table 1) and Eq. (49) gives $T_{\text {end rad.dec. }}=2.2 \times 10^{4} T_{0}$, where $T_{0}$ is the present age of the Universe.

\section{Statistical analysis}

In our statistical analysis, we used the following astronomical data: supernovae of type Ia (SNIa) (Union 2.1
$\Omega_{\text {rad.dec }}(\mathrm{t})$
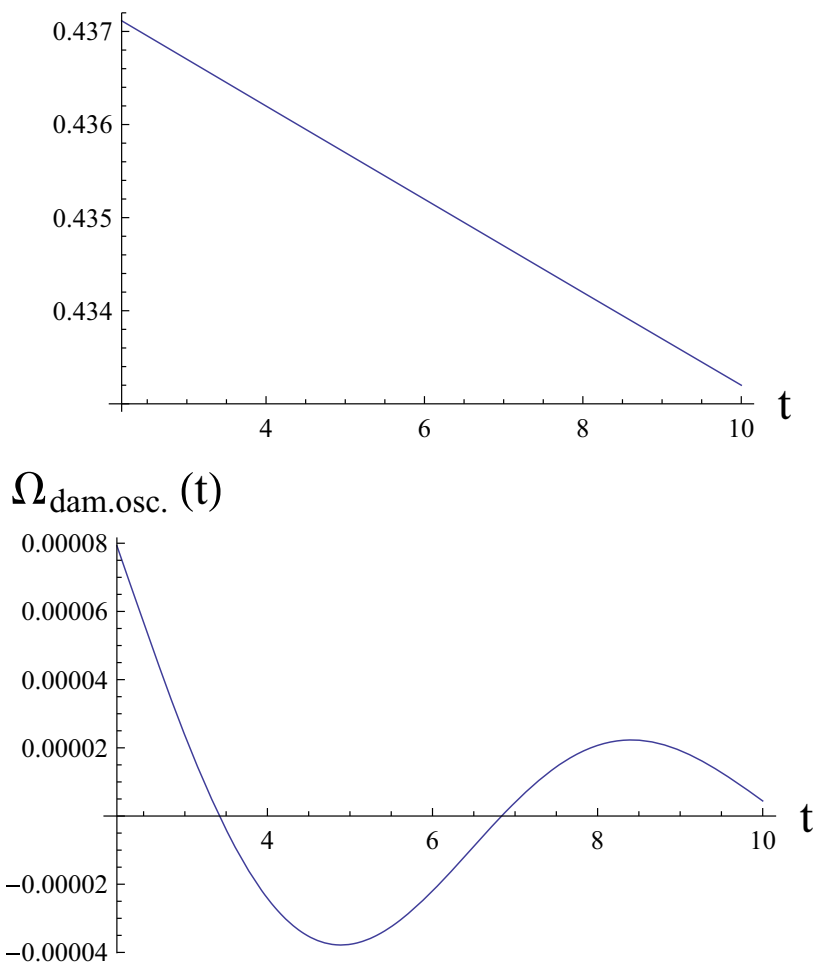

$\Omega_{\text {paw.law. }}(\mathrm{t})$

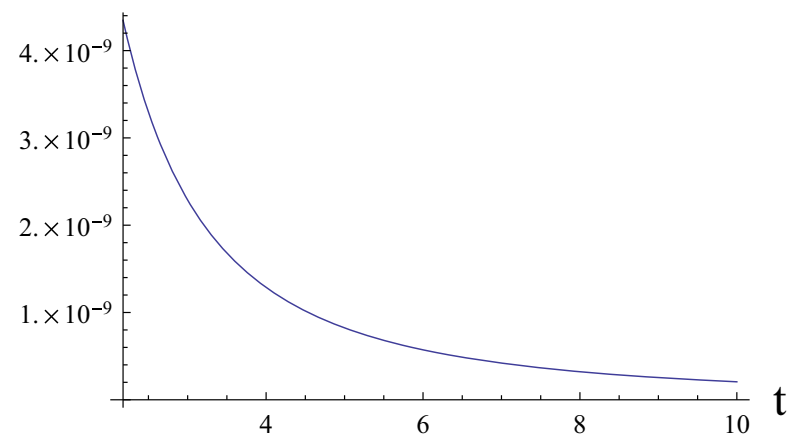

Fig. 5 The dependence $\Omega_{\text {rad.dec., }} \Omega_{\text {dam.osc., }} \Omega_{\text {pow.law with respect to }}$ the cosmological time $t$ for the best fit value of model parameter (see Table 1). The cosmological time $t$ is expressed in $\frac{\mathrm{s} \times \mathrm{Mpc}}{100 \mathrm{~km}}$. In these units, the present epoch is for $t=1.41 \frac{\mathrm{s} \times \mathrm{Mpc}}{100 \mathrm{~km}}$. Let us note that while the density parameters do not change practically during the cosmic evolution for the cases shown in the upper and middle panels, the density parameters are lowered by many orders of magnitude for the case presented in the lower panel [20]

dataset [55]), BAO data (Sloan Digital Sky Survey Release 7 (SDSS DR7) dataset at $z=0.275$ [56], 6dF Galaxy Redshift Survey measurements at redshift $z=0.1$ [57], and WiggleZ measurements at redshift $z=0.44,0.60,0.73$ [58]), measurements of the Hubble parameter $H(z)$ of galaxies [59-61], the Alcock-Paczynski test (AP) [62,63] (data from [64-72].) and measurements of CMB by Planck 


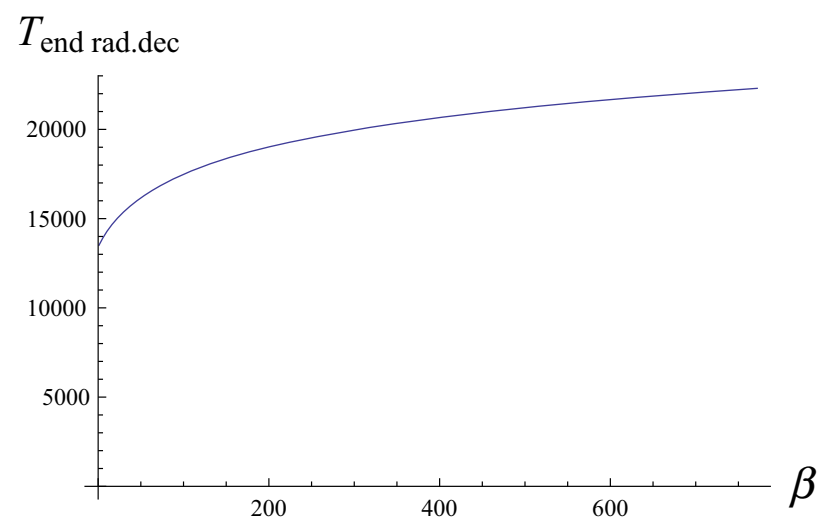

Fig. 6 A diagram presents a dependence $T_{\text {end rad.dec. }}(\beta)$ given by Eq. (49) for $\beta>29$. For illustration we put the best fit value of $\Gamma_{0}$ (see Table 1). The values of $T_{\text {end rad.dec. }}$ are expressed in terms of the present age of the Universe $T_{0}$

[73]. The equation for the likelihood function is given by

$L_{\text {tot }}=L_{\mathrm{SNIa}} L_{\mathrm{BAO}} L_{\mathrm{AP}} L_{H(z)} L_{\mathrm{CMB}}$.

The likelihood function for SNIa has the form

$L_{\mathrm{SNIa}}=\exp \left[-\frac{1}{2}\left[A-B^{2} / C+\log (C /(2 \pi))\right]\right]$,

where $A=\left(\mu^{\text {obs }}-\mu^{\text {th }}\right) \mathbb{C}^{-1}\left(\mu^{\text {obs }}-\mu^{\text {th }}\right), B=\mathbb{C}^{-1}\left(\mu^{\text {obs }}-\right.$ $\left.\mu^{\text {th }}\right), C=\operatorname{Tr} \mathbb{C}^{-1}$ and $\mathbb{C}$ is a covariance matrix for SNIa, $\mu^{\text {obs }}$ is the observer distance modulus and $\mu^{\text {th }}$ is the theoretical distance modulus.

The likelihood function for BAO is described by the equation

$L_{\mathrm{BAO}}=\exp \left[-\frac{1}{2}\left(\mathbf{d}^{\mathrm{obs}}-\frac{r_{\mathrm{s}}\left(z_{\mathrm{d}}\right)}{D_{V}(\mathbf{z})}\right) \mathbb{C}^{-1}\left(\mathbf{d}^{\mathrm{obs}}-\frac{r_{\mathrm{s}}\left(z_{\mathrm{d}}\right)}{D_{V}(\mathbf{z})}\right)\right]$,

where $r_{\mathrm{s}}\left(z_{\mathrm{d}}\right)$ is the sound horizon at the drag epoch [74,75].

The likelihood function

$L_{H(z)}=\exp \left[-\frac{1}{2} \sum_{i=1}^{N}\left(\frac{H\left(z_{i}\right)^{\mathrm{obs}}-H\left(z_{i}\right)^{\mathrm{th}}}{\sigma_{i}}\right)^{2}\right]$

is for measurements of the Hubble parameter $H(z)$ of galaxies.

The likelihood function for AP is given by

$L_{A P(z)}=\exp \left[-\frac{1}{2} \sum_{i=1}^{N}\left(\frac{A P\left(z_{i}\right)^{\mathrm{obs}}-A P\left(z_{i}\right)^{\mathrm{th}}}{\sigma_{i}}\right)^{2}\right]$,

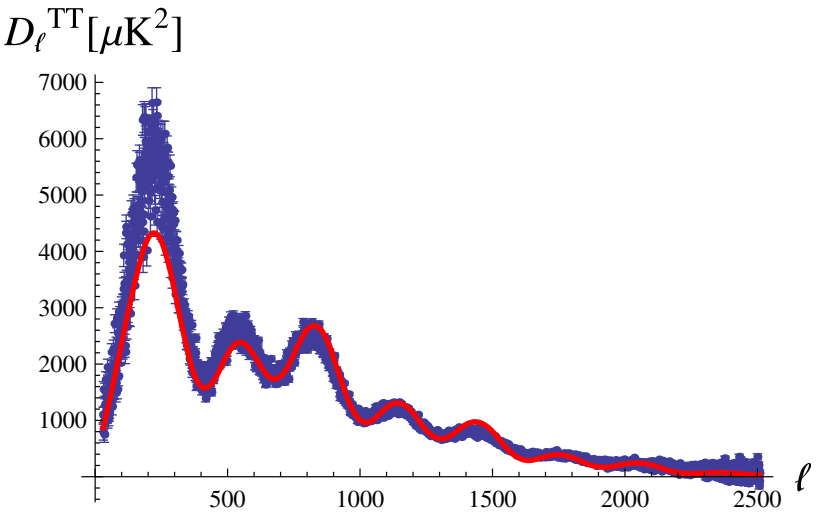

Fig. 7 Diagram of the temperature power spectrum of CMB for the best fit values (red line). The error bars from the Planck data are presented by the color blue

Table 1 The best fit and errors for the estimated model with $\alpha$ from the interval $(00.0,0.033), \Gamma_{0} / \hbar$ from the interval $\left(0.00 \frac{100 \mathrm{~km}}{\mathrm{~s} \times \mathrm{Mpc}}, 0.036 \frac{100 \mathrm{~km}}{\mathrm{~s} \times \mathrm{Mpc}}\right)$ and $\epsilon / 3 H_{0}^{2}$ from the interval $(0.00,0.0175)$. We assumed that $\Omega_{\mathrm{b}, 0}=$ $0.048468, H_{0}=67.74 \frac{\mathrm{km}}{\mathrm{s} \times \mathrm{Mpc}}$ and $\Omega_{\mathrm{m}, 0}=0.3089$. In the table, the values of $\Gamma_{0} / \hbar$ are expressed in $\frac{100 \mathrm{~km}}{\mathrm{~s} \times \mathrm{Mpc}}$. Because $\alpha=\frac{1}{1+\beta}$, the best fit value of $\beta$ parameter is equal to 799

\begin{tabular}{llll}
\hline Parameter & Best fit & $68 \% \mathrm{CL}$ & $95 \% \mathrm{CL}$ \\
\hline$\alpha$ & 0.00125 & +0.00104 & +0.01777 \\
& & -0.00125 & -0.00125 \\
$\Gamma_{0} / \hbar$ & 0.00115 & +0.00209 & +0.2123 \\
& & -0.00115 & -0.00115 \\
$\epsilon / 3 H_{0}^{2}$ & 0.0111 & +0.0064 & +0.0064 \\
& & -0.0083 & -0.0093 \\
\hline
\end{tabular}

where $A P(z)^{\text {th }} \equiv \frac{H(z)}{z} \int_{0}^{z} \frac{\mathrm{d} z^{\prime}}{H\left(z^{\prime}\right)}$ and $A P\left(z_{i}\right)^{\text {obs }}$ are observational data. The likelihood function for $\mathrm{CMB}$ is given by

$L_{\mathrm{CMB}}=\exp \left[-\frac{1}{2}\left(\mathbf{x}^{\mathrm{th}}-\mathbf{x}^{\mathrm{obs}}\right) \mathbb{C}^{-1}\left(\mathbf{x}^{\mathrm{th}}-\mathbf{x}^{\mathrm{obs}}\right)\right]$,

where $\mathbb{C}$ is the covariance matrix with the errors, $\mathbf{x}$ is a vector of the acoustic scale $l_{\mathrm{A}}$, the shift parameter $R$ and $\Omega_{b} h^{2}$ where $l_{\mathrm{A}}=\frac{\pi}{r_{\mathrm{s}}\left(z^{*}\right)} c \int_{0}^{z^{*}} \frac{\mathrm{d} z^{\prime}}{H\left(z^{\prime}\right)}$ and $R=\sqrt{\Omega_{\mathrm{m}, 0} H_{0}^{2}} \int_{0}^{z^{*}} \frac{\mathrm{d} z^{\prime}}{H\left(z^{\prime}\right)}$, where $z^{*}$ is the redshift of the epoch of the recombination [74].

In this paper, we used our own code CosmoDarkBox in the estimation of the model parameters. Our code uses the Metropolis-Hastings algorithm [76,77].

In the statistical analysis, we estimated three model parameters: $\alpha=\frac{1}{1+\beta}, \Gamma_{0}, \epsilon / 3 H_{0}^{2}$. Our statistical results are completely presented in Table 1 . The diagram of the temperature power spectrum for the best fit values is presented in Fig. 7 . Therefore the radioactive type of decay gives the most effective mechanism of the decaying metastable dark energy. We estimated also that the decay half life time $T_{1 / 2}$ of dark energy is equal to $8503 \mathrm{Gyr} \approx 616 \times T_{0}$. 


\section{Discussion and conclusions}

The main aim of this paper was to study the implication of the derived form of the running dark energy. In our approach the formula for the parametrization of this dark energy is derived directly from quantum mechanics rather than being postulated in a phenomenological way. The evolution scenario of dark energy contains three different phases: a phase of radioactive-like decay in the early Universe, a phase of damping oscillations and finally a phase of the power-law type of decay.

We investigated the cosmological evolution caused by such a variability of dark energy and matter. The dynamics of the model is governed by a cosmological dynamical system with an interacting term because the energy-momentum tensor is not conserved in this case.

Using results of the investigation of variability of dark energy with the cosmological time, we analyzed the issue of whether the problem of the cosmological constant could be solved within the considered model based on the assumption that the decay process of the dark energy is the quantum decay process having the same form as the decay process of the unstable quantum systems or not. For simplicity it was assumed that this decay process is determined by Eq. (4) with the distribution of the energy density $\omega(E)$ in the unstable quantum state having the Breit-Wigner form $\omega(E)=\omega_{\mathrm{BW}}(E)$. We show that within such a model dark energy decays and then the canonical scaling law for cold dark matter $a^{-3}$ should be modified. Unfortunately, from our analysis it follows that within the considered model, where $\omega(E)=\omega_{\mathrm{BW}}(E)$ and the assumptions leading to estimations presented in Table 1 are used, there is a very small difference between $\rho_{\mathrm{DE}}(0)$ and $\rho_{\mathrm{DE}}\left(T_{0}\right)$, which cannot be considered as a solution of the cosmological constant problem. On the other hand one cannot exclude that $\omega(E)$ has such a form as will lead by (4), for such a decay law, to $\rho_{\mathrm{DE}}(0) \gg \rho_{\mathrm{DE}}\left(T_{0}\right)$ for suitably chosen parameters of the model.

Using astronomical data we tested the model and see that it is in good agreement with the data. Our estimation also shows that the fraction of all components of the dynamical dark energy in the whole dark energy is larger than the contribution of the cosmological constant term.

In our model it is calculated that the $\Lambda$ term has a dynamical nature as a consequence of a decaying of the dark energy. In consequence the conservation of the energy-momentum tensor (EMT) is violated. Recently, Josset and Perez [51] have demonstrated the model in which the violation of EMT can be achieved in the context of the unimodular gravity and how it leads to the emergence of the effective cosmological constant in Einstein's equations. In our approach the violation of the conservation of EMT is rather a consequence of the quantum mechanical nature of the metastable vacuum, rather than a modification of the gravity theory.
In our approach the concrete form of the decaying dark energy is derived directly from a quantum mechanical consideration of unstable states. We obtain a more complex form of decaying dark energy in which we have found a radioactive type of its decay. We also estimated the model parameters as well as fractions of three different forms of decaying: radioactive type, damping oscillating type and power-law type. From the astronomical data we see that the radioactive type of decay is favored and $44 \%$ of the energy budget of the Universe corresponds with a radioactive-like decay.

In our paper we investigate the second way of the decay of dark energy into dark matter from the three different ways of dark energy decay considered by Shafieloo et al. [6]. They proposed a class of metastable dark energy models in which dark energy decays according to the radioactive law. They assumed a phenomenological form of the decay, studying observational constraints for the cosmological model. In our paper, it is derived directly from quantum mechanics. Our results are complementary to their results because they justify the phenomenological choice of the exponential decay as a major mechanism of dark energy decay. Moreover, our calculation of the decay half life is in agreement with Shafieloo et al.'s calculation. We see that the radioactive-like decay dominates up to $2.2 \times 10^{4} T_{0}$. Our calculations show that the radioactive-like decay has only an intermediate character and will be replaced in the future evolution of the Universe by an oscillation decay and then decay of $1 / t^{2}$ type.

One of the differences between our approach and the theory developed by Shafieloo et al. is that they consider only decay of the dark energy into dark constituents assuming that the decay rate $\Gamma$ of the dark energy is constant and depends only on its internal composition. The latter assumption is approximately true only if one considers decay processes as classical physics processes. The detailed analysis of decay processes of unstable quantum systems shows that the basic principles of the quantum theory do not allow them to be described by an exponential decay law at very late times as well as at initial stage of the decay process (see, e.g., [5] and the references therein, or [78]) and that the decay law can be described by the exponentially decreasing function of time only at "canonical decay regime" of the decay process, that is, at intermediate times (at times longer than the initial stage of the evolution of the unstable quantum system and shorter than the cross-over time $T$ ). These properties of quantum decay processes mean that in general the decay rate cannot be constant in time, $\Gamma=\Gamma(t) \neq$ const (see, e.g., $[32,37,42,78]$ ), and at the "canonical decay" stage $\Gamma(t) \simeq \Gamma_{0}$, to a very good approximation.

These properties of the decay rate were used in our paper. The advantage of the use of the decay rate following from the quantum properties of the decaying systems is that such an approach allows one to describe correctly the initial stage of the dark energy decay process, and at very late times. 
It is impossible to realize this within the approach used by Shafieloo et al. Moreover, the use of $\Gamma=$ const may lead to the results which need not be correct. The example of such a situation is the analysis performed in Appendix A, Section A1, of Ref. [6], where the authors considered the case $\Gamma t \ll 1$ and then applied the results obtained within such an assumption for the analysis of properties of their Model I. Namely, there are many reasons for drawing the conclusion that the decay of the dark energy must be a quantum decay process (see the discussion in Sect. 1) and that it cannot be a classical physics process. So when one wants to describe the early stage of the decay process of the dark energy, which mathematically can be expressed by the assumption that $\Gamma t \ll 1$ one should not use a relation of the type (1) but the relation

$\rho_{\mathrm{DE}}(t)=\rho_{\mathrm{DE}}(0)|A(t)|^{2}$,

resulting from the quantum mechanical treatment of the decay process. Instead of considering the relation of this type, the authors of [6] used Eq. (1), which leads to Eq. (A1) in [6] for $\Gamma t \ll 1$, that is, to

$\rho_{\mathrm{DE}}=\epsilon_{0} e^{-\Gamma t} \simeq \epsilon_{0}(1-\Gamma t)$

( $\epsilon_{0}$ is defined in [6]), which is mathematically correct but it is not correct when one considers the decay of the dark energy as a quantum process. In the case of a quantum decay process one should use a relation of the type (56) and the approximate form of $|A(t)|^{2}$ for very short times. In such a case (see, e.g. [5,32])

$|A(t)|^{2} \simeq 1-d^{2} t^{2}$, for $t \rightarrow 0$

where $d=$ const and it does not depend on $\Gamma$. Therefore we should have

$\rho_{\mathrm{DE}}(t) \simeq \rho_{\mathrm{DE}}(0)\left(1-d^{2} t^{2}\right)$, for $t \rightarrow 0$,

for short times $t$, when the decay of the dark energy is a quantum decay process. The difference between Eqs. (57) (i.e., (A1) in [6]) and (59) is dramatic (the use $\epsilon_{0}$ in (57) and $\rho_{\mathrm{DE}}(0)$ in (59) is not the point). The problem is that the authors of [6]) use their result (A1) (that is, Eq. (57)) in Eq. (A2) and then all considerations related to their Model I in Section A I of Appendix A are founded on Eqs. (A1) and (A2). This means that the conclusions drawn in [6] (based on the analysis performed in Sect. A I of Appendix A) may not reflect real properties of the decaying dark energy. It should be noted that our analysis performed in this paper is free of this defect.

Note also that Shafieloo et al. [6] considered only the decays of the dark energy into dark components: dark mat- ter and dark radiation, whereas we consider the general case (that is, in our approach the decay of the dark energy into a visible baryonic matter is also admissible, which cannot be excluded in the light of the recently reported discovery of baryonic spindles linking galaxies [79-81]).

Open Access This article is distributed under the terms of the Creative Commons Attribution 4.0 International License (http://creativecomm ons.org/licenses/by/4.0/), which permits unrestricted use, distribution, and reproduction in any medium, provided you give appropriate credit to the original author(s) and the source, provide a link to the Creative Commons license, and indicate if changes were made.

Funded by SCOAP $^{3}$.

\section{References}

1. L.M. Krauss, J. Dent, Phys. Rev. Lett. 100, 171301 (2008). https:// doi.org/10.1103/PhysRevLett.100.171301. arXiv:0711.1821

2. N.S. Krylov, V.A. Fock, Zh. Eksp, Teor. Fiz. 17, 93 (1947) (in Russian)

3. V.A. Fock, Fundamentals of Quantum Mechanics (Mir Publishers, Moscow, 1978)

4. L.A. Khalfin, Zh. Eksp. Teor. Fiz. (USSR) 33, 1371 (1957) (in Russian). [Sov. Phys. JETP 6, 1053 (1958)]

5. L. Fonda, G.C. Ghirardi, A. Rimini, Rep. Prog. Phys. 41, 587 (1978). https://doi.org/10.1088/0034-4885/41/4/003

6. A. Shafieloo, D.K. Hazra, V. Sahni, A.A. Starobinsky, Metastable dark energy with radioactive-like decay. Mon. Not. R. Astron. Soc. (2017) (in press). https://doi.org/10.1093/mnras/stx2481. arXiv: 1610.05192

7. J. Polchinski, in The Quantum Structure of Space and Time: Proceedings of the 23rd Solvay Conference on Physics, Brussels, Belgium, 1-3 December 2005 (2006), pp. 216-236. arXiv:hep-th/0603249

8. S. Weinberg, Rev. Mod. Phys. 61, 1 (1989). https://doi.org/10. 1103/RevModPhys.61.1

9. S.M. Carroll, W.H. Press, E.L. Turner, Ann. Rev. Astron. Astrophys. 30, 499 (1992). https://doi.org/10.1146/annurev.aa.30. 090192.002435

10. A.D. Dolgov, in Phase transitions in cosmology. Proceedings, 4th Cosmology Colloquium, Euroconference, Paris, France, June 49, 1997 (1997), pp. 161-175. http://alice.cern.ch/format/showfull? sysnb=0254991. arXiv:astro-ph/9708045

11. V. Sahni, A.A. Starobinsky, Int. J. Mod. Phys. D 9, 373 (2000). arXiv:astro-ph/9904398

12. N. Straumann, Eur. J. Phys. 20, 419 (1999). https://doi.org/10. 1088/0143-0807/20/6/307. arXiv:astro-ph/9908342

13. S. Weinberg, in Sources and detection of dark matter and dark energy in the universe. Proceedings, 4th International Symposium, DM 2000, Marina del Rey, USA, February 23-25, 2000 (2000), pp. 18-26. arXiv:astro-ph/0005265

14. S.M. Carroll, Living Rev. Relativ. 4, 1 (2001). https://doi.org/10. 12942/lrr-2001-1. arXiv:astro-ph/0004075

15. S.E. Rugh, H. Zinkernagel, Stud. Hist. Philos. Sci. B33, 663 (2002). https://doi.org/10.1016/S1355-2198(02)00033-3. arXiv:hep-th/0012253

16. T. Padmanabhan, Phys. Rep. 380, 235 (2003). https://doi.org/10. 1016/S0370-1573(03)00120-0. arXiv:hep-th/0212290

17. J. Yokoyama, in Proceedings, 12th Workshop on General Relativity and Gravitation (JGRG12): Tokyo, Japan, November 25-28, 2002 (2003). arXiv:gr-qc/0305068 
18. S. Sarkar, Curr. Sci. 88, 2120 (2005)

19. E.J. Copeland, M. Sami, S. Tsujikawa, Int. J. Mod. Phys. D 15, 1753 (2006). https://doi.org/10.1142/S021827180600942X. arXiv:hep-th/0603057

20. M. Szydlowski, Phys. Rev. D 91(12), 123538 (2015). https://doi. org/10.1103/PhysRevD.91.123538. arXiv:1502.04737

21. A. Patrascioiu, Phys. Rev. D 24, 496 (1981). https://doi.org/10. 1103/PhysRevD.24.496

22. S.R. Coleman, Phys. Rev. D 15, 2929 (1977). https://doi.org/10. 1103/PhysRevD.15.2929. https://doi.org/10.1103/PhysRevD.16. 1248. [Erratum: Phys. Rev. D 16, 1248 (1977)]

23. C.G. Callan Jr., S.R. Coleman, Phys. Rev. D 16, 1762 (1977). https://doi.org/10.1103/PhysRevD.16.1762

24. S.R. Coleman, F. De Luccia, Phys. Rev. D 21, 3305 (1980). https:// doi.org/10.1103/PhysRevD.21.3305

25. S. Weinberg, The Quantum Theory of Fields, vol. 2, Modern Applications (Cambridge University Press, Cambridge, 1995)

26. R.G. Landim, E. Abdalla, Phys. Lett. B 764, 271 (2017). https:// doi.org/10.1016/j.physletb.2016.11.044. arXiv:1611.00428

27. E. Di Valentino, E.V. Linder, A. Melchiorri, A Vacuum Phase Transition Solves $H_{0}$ Tension (2017). arXiv:1710.02153

28. E. Rutherford, Philos. Mag. Ser. 5 XLIX, 1 (1900)

29. E. Rutherford, Philos. Mag. Ser. 5 XLIX, 161 (1900)

30. E. Rutherford, F. Soddy, Philos. Mag. Ser. 6 IV, 370 (1902)

31. E. Rutherford, F. Soddy, Philos. Mag. Ser. 6 IV, 569 (1902)

32. K. Urbanowski, Phys. Rev. A 50, 2847 (1994). https://doi.org/10. 1103/PhysRevA.50.2847

33. N.G. Kelkar, M. Nowakowski, J. Phys. A 43, 385308 (2010). https://doi.org/10.1088/1751-8113/43/38/385308. arXiv: 1008.3917

34. J. Martorell, J.G. Muga, D.W.L. Sprung, in Time in Quantum Mechanics vol. 2, ed. by G. Muga, A. Ruschhaupt, A. del Campo, Lect. Notes Phys. 789 (Springer, Berlin, 2009) pp. 329-275

35. E. Torrontegui, J.G. Muga, J. Martorell, D.W.L. Sprung, Adv. Quantum Chem. 60, 485 (2010)

36. G. Garcia-Calderon, R. Romo, J. Villavicencio, Phys. Rev. B 76, 035340 (2007). https://doi.org/10.1103/PhysRevB.76.035340

37. F. Giraldi, Eur. Phys. J. D 69, 5 (2015)

38. F. Giacosa, Found. Phys. 42, 1262 (2012). https://doi.org/10.1007/ s10701-012-9667-3. arXiv:1110.5923

39. M.L. Goldberger, K.M. Watson, Collision theory (Wiley, New York, 1964)

40. K.M. Sluis, E.A. Gislason, Phys. Rev. A 43, 4581 (1991). https:// doi.org/10.1103/PhysRevA.43.4581

41. K. Urbanowski, Eur. Phys. J. C 58, 151 (2008). https://doi.org/10. 1140/epjc/s10052-008-0725-0. arXiv:hep-ph/0610384

42. K. Urbanowski, Cent. Eur. J. Phys. 7, 696 (2009)

43. K. Urbanowski, Eur. Phys. J. D 54, 25 (2009). https://doi.org/10. 1140/epjd/e2009-00165-x. arXiv:0803.3188

44. G. Breit, E. Wigner, Phys. Rev. 49, 519 (1936). https://doi.org/10. 1103/PhysRev.49.519

45. F.W.J. Olver, D.W. Lozier, R.F. Boisvert, C.W. Clark (eds.), NIST Handbook of Mathematical Functions, National Institute of Standards and Technology (Cambridge University Press, Cambridge, 2010)

46. M. Abramowitz, I.A. Stegun (eds.), Handbook of Mathematical Functions, National Bureau of Standards, Appl. Math. Ser. No. 55 (U.S. G.P.O., Washington, D.C., 1964)

47. K. Urbanowski, Phys. Rev. Lett. 107, 209001 (2011). https://doi. org/10.1103/PhysRevLett.107.209001. arXiv:1111.3912

48. K. Urbanowski, M. Szydlowski, AIP Conf. Proc. 1514, 143 (2012). https://doi.org/10.1063/1.4791743. arXiv:1304.2796

49. M. Szydlowski, A. Stachowski, K. Urbanowski, J. Phys. Conf. Ser. 626, 012033 (2015). https://doi.org/10.1088/1742-6596/626/ 1/012033. arXiv:1502.04471
50. K. Urbanowski, Theor. Math. Phys. 190, 458 (2017). https://doi. org/10.1134/S0040577917030151. arXiv:1609.03382

51. T. Josset, A. Perez, D. Sudarsky, Phys. Rev. Lett. 118, 021102 (2017). https://doi.org/10.1103/PhysRevLett.118.021102. arXiv: 1604.04183

52. C.B. Chiu, E.C.G. Sudarshan, B. Misra, Phys. Rev. D 16, 520 (1977). https://doi.org/10.1103/PhysRevD.16.520

53. M. Szydlowski, A. Stachowski, JCAP 1510, 066 (2015). https:// doi.org/10.1088/1475-7516/2015/10/066. arXiv:1507.02114

54. M. Szydlowski, A. Stachowski, Phys. Dark Univ. 15, 96 (2017). https://doi.org/10.1016/j.dark.2017.01.002. arXiv:1508.05637

55. N. Suzuki et al., Astrophys. J. 746, 85 (2012). https://doi.org/10. 1088/0004-637X/746/1/85. arXiv: 1105.3470

56. W.J. Percival et al., Mon. Not. R. Astron. Soc. 401, 2148 (2010). https://doi.org/10.1111/j.1365-2966.2009.15812.x. arXiv:0907.1660

57. F. Beutler, C. Blake, M. Colless, D.H. Jones, L. Staveley-Smith, L. Campbell, Q. Parker, W. Saunders, F. Watson, Mon. Not. R. Astron. Soc. 416, 3017 (2011). https://doi.org/10.1111/j.1365-2966.2011. 19250.x. arXiv:1106.3366

58. C. Blake et al., Mon. Not. R. Astron. Soc. 425, 405 (2012). https:// doi.org/10.1111/j.1365-2966.2012.21473.x. arXiv:1204.3674

59. J. Simon, L. Verde, R. Jimenez, Phys. Rev. D 71, 123001 (2005). https://doi.org/10.1103/PhysRevD.71.123001. arXiv:astro-ph/0412269

60. D. Stern, R. Jimenez, L. Verde, M. Kamionkowski, S.A. Stanford, JCAP 1002, 008 (2010). https://doi.org/10.1088/1475-7516/2010/ 02/008. arXiv:0907.3149

61. M. Moresco et al., JCAP 1208, 006 (2012). https://doi.org/10.1088/ 1475-7516/2012/08/006. arXiv:1201.3609

62. C. Alcock, B. Paczynski, Nature 281, 358 (1979). https://doi.org/ $10.1038 / 281358 \mathrm{a} 0$

63. M. Lopez-Corredoira, Astrophys. J. 781(2), 96 (2014). https://doi. org/10.1088/0004-637X/781/2/96. arXiv:1312.0003

64. P.M. Sutter, G. Lavaux, B.D. Wandelt, D.H. Weinberg, Astrophys. J. 761, 187 (2012). https://doi.org/10.1088/0004-637X/761/2/187. arXiv: 1208.1058

65. C. Blake et al., Mon. Not. R. Astron. Soc. 418, 1725 (2011). https:// doi.org/10.1111/j.1365-2966.2011.19606.x. arXiv:1108.2637

66. N.P. Ross et al., Mon. Not. R. Astron. Soc. 381, 573 (2007). https://doi.org/10.1111/j.1365-2966.2007.12289.x. arXiv:astro-ph/0612400

67. C. Marinoni, A. Buzzi, Nature 468(7323), 539 (2010). https://doi. org/10.1038/nature09577

68. J. da Angela, P.J. Outram, T. Shanks, Mon. Not. R. Astron. Soc. 361, 879 (2005). https://doi.org/10.1111/j.1365-2966.2005. 09212.x. arXiv:astro-ph/0505469

69. P.J. Outram, T. Shanks, B.J. Boyle, S.M. Croom, F. Hoyle, N.S. Loaring, L. Miller, R.J. Smith, Mon. Not. R. Astron. Soc. 348, 745 (2004). https://doi.org/10.1111/j.1365-2966.2004. 07348.x. arXiv:astro-ph/0310873

70. L. Anderson et al., Mon. Not. R. Astron. Soc. 427(4), 3435 (2013). https://doi.org/10.1111/j.1365-2966.2012.22066.x. arXiv:1203.6594

71. I. Paris et al., Astron. Astrophys. 548, A66 (2012). https://doi.org/ 10.1051/0004-6361/201220142. arXiv:1210.5166

72. D.P. Schneider et al., Astron. J. 139, 2360 (2010). https://doi.org/ 10.1088/0004-6256/139/6/2360. arXiv:1004.1167

73. P.A.R. Ade et al., Astron. Astrophys. 594, A14 (2016). https://doi. org/10.1051/0004-6361/201525814. arXiv:1502.01590

74. W. Hu, N. Sugiyama, Astrophys. J. 471, 542 (1996). https://doi. org/10.1086/177989. arXiv:astro-ph/9510117

75. D.J. Eisenstein, W. Hu, Astrophys. J. 496, 605 (1998). https://doi. org/10.1086/305424. arXiv:astro-ph/9709112 
76. N. Metropolis, A.W. Rosenbluth, M.N. Rosenbluth, A.H. Teller, E. Teller, J. Chem. Phys. 21, 1087 (1953). https://doi.org/10.1063/1. 1699114

77. W.K. Hastings, Biometrika 57, 97 (1970). https://doi.org/10.1093/ biomet/57.1.97

78. K. Urbanowski, Eur. Phys. J. D 71, 118 (2017). https://doi.org/10. 1140/epjd/e2017-70666-0
79. H. Tanimura, G. Hinshaw, I.G. McCarthy, L. Van Waerbeke, Y.Z. Ma, A. Mead, A. Hojjati, T. Troester, A Search for Warm/Hot Gas Filaments Between Pairs of SDSS Luminous Red Galaxies (2017). arXiv:1709.05024

80. A. de Graaff, Y.C. Cai, C. Heymans, J.A. Peacock, Missing baryons in the cosmic web revealed by the Sunyaev-Zel'dovich effect (2017). arXiv: 1709.10378

81. I. Fernini, Astron. Phys. Wkly. News 7, 41 (2017) 